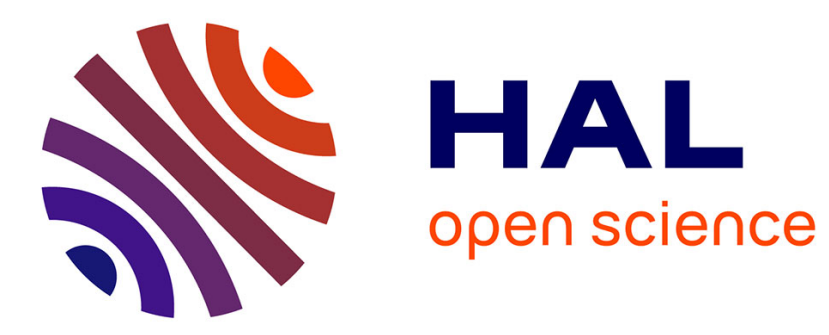

\title{
Reptation of stars
}

P.-G. de Gennes

\section{To cite this version:}

P.-G. de Gennes. Reptation of stars. Journal de Physique, 1975, 36 (12), pp.1199-1203. 10.1051/jphys:0197500360120119900 . jpa-00208365

\section{HAL Id: jpa-00208365 https://hal.science/jpa-00208365}

Submitted on 1 Jan 1975

HAL is a multi-disciplinary open access archive for the deposit and dissemination of scientific research documents, whether they are published or not. The documents may come from teaching and research institutions in France or abroad, or from public or private research centers.
L'archive ouverte pluridisciplinaire HAL, est destinée au dépôt et à la diffusion de documents scientifiques de niveau recherche, publiés ou non, émanant des établissements d'enseignement et de recherche français ou étrangers, des laboratoires publics ou privés. 
Classification

Physics Abstracts

1.660

\title{
REPTATION OF STARS
}

\author{
P. G. DE GENNES \\ Collège de France, 75231 Paris Cedex 05, France
}

(Reçu le 4 juillet 1975, accepté le 30 juillet 1975)

\begin{abstract}
Résumé. - On discute le mouvement brownien d'un polymère flexible en forme d'étoile, placé dans un réseau fixe (gel). On prédit : $1^{\circ}$ un temps de renouvellement de la conformation globale $T_{\mathrm{r}}$ qui croît exponentiellement avec la masse $M$ - donc beaucoup plus long que pour une chaîne linéaire, $2^{\circ}$ une relaxation logarithmique des tensions mécaniques.

Nous ne savons pas, à l'heure actuelle, si ces propriétés doivent se retrouver pour des étoiles en phase fondue.
\end{abstract}

Abstract. - We consider the stochastic motions of a star-shaped flexible polymer trapped inside a fixed network. We predict : $1^{\circ}$ a renewal time $T_{\mathrm{r}}$ for the star conformations which increases exponentially with the molecular mass $M$, and $2^{\circ}$ a logarithmic relaxation of stress.

We do not know whether these properties still hold for a homogeneous melt of stars, or not.

1. Introduction. - The mechanical behaviour of dilute flexible chains trapped inside a polymer network has been studied by Ferry and coworkers $[1,2]$. The data suggest that the renewal time for the conformations of the mobile chains increases with their molecular mass $M$ like a certain power $M^{x}$, with $x$ values ranging between 3 and 3.5. A comparatively simple model, based on the reptation concept [3], predicted $x=3$.

In the present note, we extend the discussion of references [3] to the case of branched molecules trapped in a network. We restrict our attention to the simple case of a star with $N$ monomers and $f$ prongs (each of them carrying $N / f$ monomers) converging to one center $C$. There exists an extensive literature on the synthesis and on the physical properties of these stars $[4,5]$. We shall consider here the reptation properties of an ideal star, assuming that :

i) the length of the branches is very long when compared to the mesh of the surrounding network

ii) the central module $\mathrm{C}$ is point like, and

iii) that excluded volume effects within the star are negligible.

Even after these simplifications, we are left with a rather complex stochastic problem : the following discussion is very qualitative, but it does show some remarkable features which are probably modelindependent.

2. Quenching of reptation. - Consider for instance the star with three branches $(f=3)$ shown in figure 1 .
The three ends are the points $E_{1}, E_{2}, E_{3}$. If we had only a linear chain $\left(E_{2} C E_{3}\right)$ it would move by snakelike motions inside a certain tube defined by the surrounding gel; but the extra branch $\left(\mathrm{CE}_{1}\right)$ blocks this reptation very strongly. Let us assume that, with an external force, we have pulled the chain $\left(\mathrm{E}_{2} \mathrm{CE}_{3}\right)$ to the right on figure 1 , the center moving from $\mathrm{C}_{0}$ to $\mathrm{C}$. The distance $\mathrm{C}_{0} \mathrm{C}$ corresponds to a certain multiple $p$ of the mesh size. This process reduced the entropy of the chain $\left(\mathrm{CE}_{1}\right)$ by an amount $p \ln z$ where $z$ is the number of gates surrounding one unit cell in the network $\left({ }^{1}\right)$. As soon as $p$ exceeds a few units this entropy defect is very large, and there is a huge elastic force tending to bring the center back to its original position.

Let us fix $p$ at a value for which the entropy is not too large (e.g. $p=1$ ) and maintain the center of the star at point $\mathrm{C}$ by some external means. How long will it take for the chain $\left(\mathrm{CE}_{1}\right)$ to return to equilibrium ? This requires that the original conformation $\left(\mathrm{CE}_{10}\right)$ be transformed onto a completely different conformation $\left(\mathrm{CE}_{1}^{\prime}\right)$ such as the one shown on figure 1.

If $\left(\mathrm{CE}_{1}\right)$ was not attached to anything, this renewal would require a time of order $(N / f)^{3}$ according to reference [3]. But here, one end (C) is fixed : the only way to construct $C_{1}^{\prime}$ from $C_{1}$ is to have $E_{1}$ come back to $C$, following exactly the original tube which surrounded $\left(\mathrm{CE}_{1}\right)$. After $\mathrm{E}_{1}$ has retraced its steps to $\mathrm{C}$, it will be able to start generating a new tube $\left(\mathrm{CE}_{1}^{\prime}\right)$.

( $\left.{ }^{1}\right)$ We use units where Boltzmann's constant is equal to unity. 


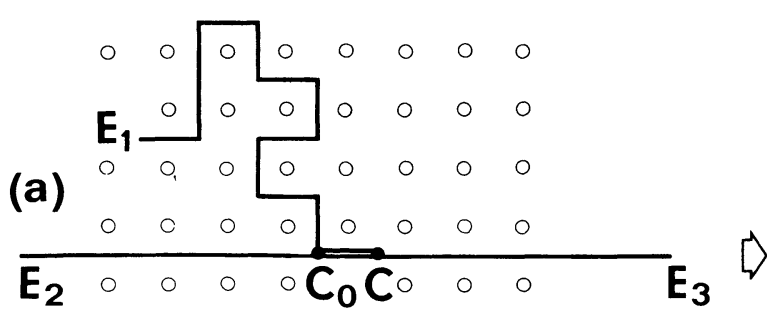

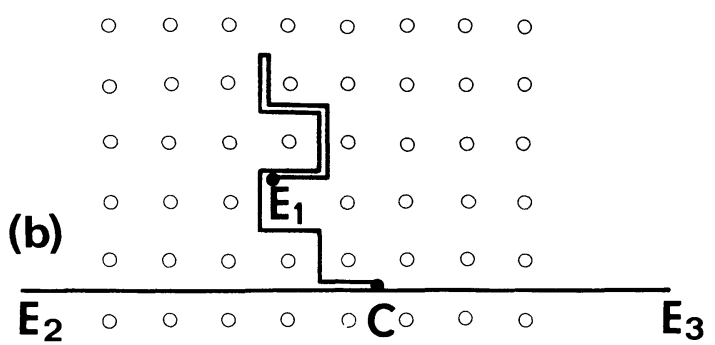

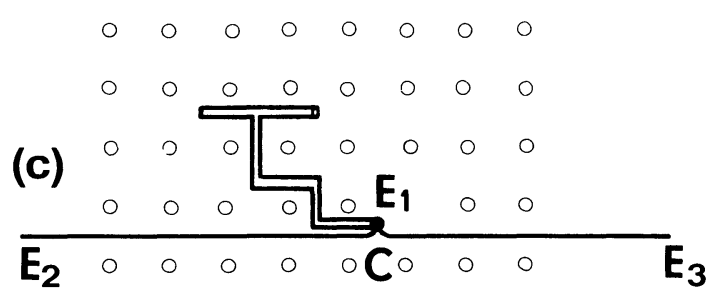

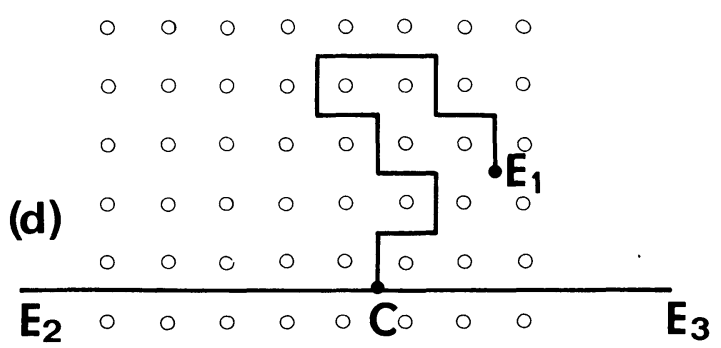

FIG. 1. - A star in a gel. The units of the network are represented here as a regular array of obstacles (white dots) which cannot be crossed. $a$ ) The main chain of the star $\left(\mathrm{E}_{2} \mathrm{E}_{3}\right)$ has been pulled to the right, the attachment point moving from $\mathrm{C}_{0}$ to $\mathrm{C} . b$ ), $c$ ) In order to create a completely new conformation, the lateral chain $\left(\mathrm{CE}_{1}\right)$ must first fold on itself. $d$ ) After reaching $\mathrm{C}$, the point $\mathrm{E}_{1}$ may start again and set up the new conformation.

It is only after completion of such a process that the entropy defect of the chain $\left(\mathrm{CE}_{1}\right)$ will be wiped out.

The probability of $E_{1}$ retracing its steps correctly to the center (as shown on Fig. 1) is very small. In order to discuss this probability, we describe the chain $\mathrm{CE}_{1}$ by a random walk of $S$ steps on a periodic lattice (Fig. 2). Each lattice site corresponds to a unit cell of the network, and has $z$ neighbours. (The simplest idealisation would correspond to a cubic lattice, with $z=6$.) The number $S$ is proportionnal to the number of monomers $(N / f)$ on the chain $\mathrm{CE}_{1}$

$$
S=q N / f
$$

where $q$ depends on the relative size of monomer units and network unit cells (and $f=3$ for the above example). (a)

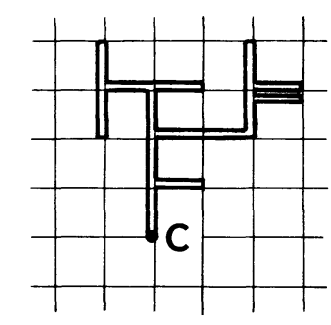

(b)

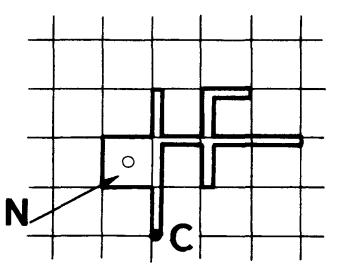

(c)
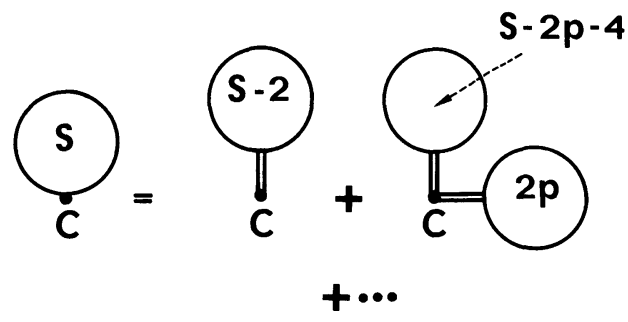

FIG. 2. - Lattice model for the folded conformations of the lateral chain $: a$ ) acceptable (tree like); $b$ ) unacceptable $:$ in this conformation the chain has gone around certain segments of the network (point $\mathrm{N}$ on the figure), and if we pull on both chain ends we cannot get the chains out of the network; $c$ ) recurrence equation for the number of trees with $S$ segments : a circle marked $S$ represents the sum of all trees with $S$ segments.

The total number of paths with $S$ steps starting from one given point $\mathrm{C}$ on the lattice is $z^{S}$. We want to count the fraction $P$ of these paths for which

i) the end point is at the origin $\left({ }^{2}\right)$, and

ii) the path is topologically equivalent to zero i.e. it has the tree structure shown on figure $2 b$.

The quantity $P$ is calculated in the appendix, and has the form

$$
P=A(z \ln 2)^{-S / 2}=\tilde{A} \mathrm{e}^{-\alpha S}
$$

where $A$ and $\tilde{A}$ are only weakly dependent on $S$. Taking $z=6$ the factor $\alpha$ is equal to 0.72 .

We may assume that the average relaxation rate $1 / \theta$ for the chain $C_{1}$ from an initial state $C_{10}$ to a final state $\mathrm{CE}_{1}^{\prime}$ (as shown on figure 1) is essentially proportionnal to $P(S)$

$$
\frac{1}{\theta}=\frac{1}{\theta_{1}} P(S)=\frac{1}{\tilde{\theta}} \mathrm{e}^{-\alpha S}=\frac{1}{\tilde{\theta}} \mathrm{e}^{-\tilde{\alpha} N / \mathcal{f}}
$$

where $\theta_{1}$ and $\tilde{\theta}$ are weak $\left({ }^{3}\right)$ (non exponential) functions of $S$, and $\tilde{\alpha}=q \alpha$.

$\left(^{2}\right)$ For simplicity we assume $S$ even.

$\left({ }^{3}\right)$ Reptation arguments suggest $1 / \theta_{1} \sim S^{3}$. 
Knowing the relaxation rate (3) we can now return to the complete problem of star motion, considering the star as a chain $\left(E_{2} C E_{3}\right)$ hindered by a lateral group $\left(\mathrm{CE}_{1}\right)$. Central to the reptation model is the motion of the mobility of a chain inside its own tube $\mu_{\mathrm{t}}$. For a chain without lateral groups, $\mu_{\mathrm{t}}$ is inversely proportionnal to chain length $\left({ }^{3}\right)$. Here, however, the tube mobility will be limited by the lateral groups, and proportionnal to their jump frequency $1 / \theta$.

Thus we expect

$$
\mu_{\mathrm{t}}=\tilde{\mu} \mathrm{e}^{-\tilde{\alpha} N / f}
$$

where all non exponential factors (depending on $N$ and $f$ ) have been included in $\tilde{\mu}$.

All the dynamical constants characteristic of long time behaviour for the star can be derived from the tube mobility as in reference [3] : they will all show the same exponential factor. For instance, the renewal time for the conformations of the whole star will be of the form

$$
T_{\mathrm{r}}=\widetilde{T}_{\mathrm{r}} \mathrm{e}^{\tilde{\alpha} N / f}
$$

and the self diffusion constant will be proportionnal to $\mathrm{e}^{-\tilde{\alpha} N / f}$. Thus we conclude that reptation is essentially quenched for a star trapped in a network.

3. Mechanical relaxation. - The relaxation time $T_{\mathrm{r}}$ is exponentially large, and may be difficult to detect directly. The behaviour at frequencies somewhat higher that $\frac{1}{T_{\mathrm{r}}}$ is also non-trivial and it is important to realize that although complete renewal of the star conformation is extremely slow, partial renewal is possible in finite times.

Let us consider a conceptual experiment where the system is at rest from $t=-\propto$ to $t=0$, and then a constant strain $e$ is imposed to the network for all later times. The stars trapped in the network are at first very distorted but they tend to relax towards a less distorted equilibrium state. After a time $t$ a number $l$ of monomers, near each extremity of the star, have relaxed by a process analog to the one illustrated in figure $1:$ the length of the portion concerned is now $l$ rather than $N / f$. The relation between $l$ and $t$ is the analog of eq. (3) relating $N / f$ and $\theta:$ we may write :

$$
l=\tilde{\alpha}^{-1} \ln (t / \tilde{\theta}) \quad\left(\theta<t<T_{\mathrm{r}}\right) .
$$

Here $\tilde{\theta}$ is an average reptation time for a chain of length $l$ (but the $l$ dependence of $\theta$ is negligible since it comes inside a logarithm). It is natural to assume that the mechanical stress $\sigma(t)$ at time $t$ will have a component proportionnal to $l(t)$

$$
\sigma(t)=e\left[E_{0}-a l(t)\right] .
$$

It would be interesting to search for this logarithmic relaxation stress in suitable systems.

In a gel without stress, if there are dangling chains (attached to the network by one end only) and if these chains are longer than the mesh size, they would also give rise to a logarithmic law of this type [6]. However, most gelation processes should not give rise to very long dangling chains : observations on gels with stars incorporated would probably be more clear cut.

4. Comparison with polymer melts. - The rheological properties of branched polymer melts have been measured in a certain number of cases [7-14], [5]. Restricting our attention to the limit of high molecular masses $M$ (strongly entangled systems) and to the linear viscosity $\eta$, we find that $\eta$ is usually increased by branching at fixed $M$. A recent study [14] suggests that for branched polyisoprene $\eta \sim \exp (M \times$ const. $)$ while for linear molecules $\eta$ is of the usual form $\eta \sim M^{a}$. It is tempting to interpret this in the following terms : the viscosity $\eta$ is the product of a relaxation time $\tau$ by an elastic modulus $E$; the latter (describing the rubber like behaviour of the system at frequencies $\omega \gg 1 / \tau)$ is controlled by the density of entanglement points, and is expected to be nearly independent of $M$ for high $M$. If the Maxwell relaxation time $\tau$ for the molten system was similar in behaviour to the renewal time $T_{\mathrm{r}}$ of the reptation problem, we would indeed be led to an exponential viscosity

$$
\eta \sim \exp \left(\frac{\tilde{\alpha}}{f} N\right)
$$

However, we wish to point out that this extrapolation is highly non trivial. Indeed, we shall now describe one possible model for molten stars, which leads to a very different behaviour, namely

$$
\eta \sim N^{3} \quad(?)
$$

In a liquid phase, any polymer molecule (linear or branched) can still be pictured as trapped in a certain tube, defined by the surrounding chains [15]. The tube concept has been extensively used (for unbranched molecules) by Edwards and coworkers [16] and by Doi [17]. In simple terms we may say that the renewal of conformations for one molecule takes place through two distinct channels :

a) reptation inside a tube which may fluctuate in position, but which maintains the same entanglements constraints all the time;

b) tube reorganisation : this corresponds to the loss of one entanglement constraint, and is shown on figure 3 : when one end of chain $\Gamma^{\prime}$ gets in the immediate vicinity of chain $\Gamma$, the relative positions of $\Gamma$ and $\Gamma^{\prime}$ may change qualitatively in a very short time. 
(a)

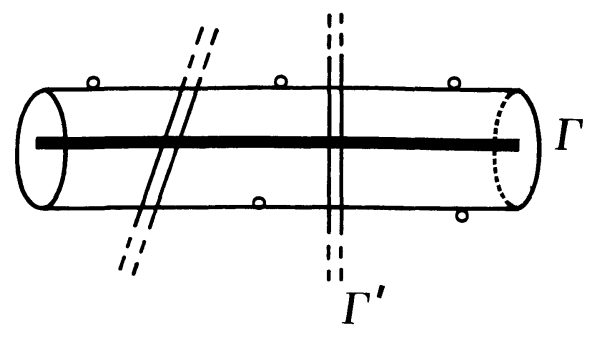

(b)

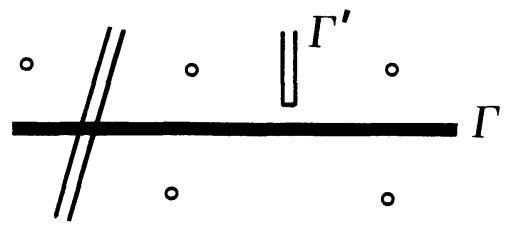

(c)

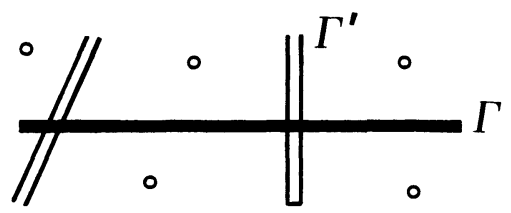

FIG. 3. - The concept of tube reorganisation : a) the chain $\Gamma$ (black) is surrounded by other chains (white) and thus trapped in a certain tube; $b$ ) one of the surrounding chains $\Gamma^{\prime}$ may have its end point close to $\Gamma ; c$ ) then it is possible for $\Gamma^{\prime}$ to go from below $\Gamma$ to above $\Gamma$ : the tube has changed its topological significance.

The model postulates that the contributions of $a$ ) and $b$ ) to the relaxation rate are roughly additive

$$
\frac{1}{T_{\mathrm{r}}}=\frac{1}{T_{\mathrm{a}}}+\frac{1}{T_{\mathrm{b}}}
$$

The essential assumption is now the following : to describe process $b$ ), we picture the tube itself as one Rouse chain [18] (branched or linear, depending on the case at hand) with one modification only : the microscopic jump frequency $W$ (the high frequency limit of the Rouse spectrum) is reduced, since only a fraction $\Phi$ of the tube sites are near the end of a neighbouring chain $\Gamma^{\prime}$, and thus susceptible of being reorganised

$$
W \rightarrow \tilde{W}=W \Phi
$$

where $\Phi$ is the relative concentration of monomers located at terminal sites : for stars with $f$ prongs,

$$
\Phi \sim \frac{f}{N}
$$

From the equations of the Rouse model we then expect a renewal rate

$$
\frac{1}{T_{\mathrm{b}}}=\text { const. } \frac{\tilde{W}}{N^{2}}=\text { const. } \frac{W}{N^{3}} .
$$

The constant in eq. (11) depends on the detailed geometry (chain [18] or $f$-pronged star [19]) but the general structure is always the same. Consider first the case of a chain. Then $\frac{1}{T_{\mathrm{a}}}$ and $\frac{1}{T_{\mathrm{b}}}$ are comparable, and the reptation laws are not qualitatively modified by tube reorganisation : this conclusion agrees with references [16-17]. On the other hand, for stars, eq. (5) predicts that $1 / T_{\mathrm{a}}$ is exponentially small. In this model the behaviour of molten stars is thus dominated by tube reorganisation : we expect $\eta \sim T_{\mathrm{r}} \sim N^{3}$ and a self diffusion coefficient of the form

$$
D=D_{0} \Phi / N=D_{0} f / N^{2}
$$

where $D_{0}$ is a microscopic constant. (We do not know of any measurements of $D$.)

5. Conclusions. - 5.1 Star reptation may appear at first sight as a highly specialised problem. However, our discussion, in spite of its qualitative nature, does give some possibly useful pictures for the motions of branched polymers. The creep properties expected for long dangling chains in a gel are interesting; they may also play a role in the long-time effects often observed in polymer adsorption on solid surfaces.

5.2 It is not clear whether reptation processes remain dominant in concentrated solutions of branched polymers (where other types of molecular rearrangement also come into play), or not. The recent data on polyisoprenes [14] suggest that they do. Earlier data on polystyrene [11], as well as the theoretical model of section 4, tend to favor the opposite conclusion. A much more detailed analysis, similar in spirit to reference [16], will be required to elucidate this point.

Acknowledgments. - The author has greatly benefited from a correspondence with Dr. W. W. Graessley, both on entanglement processes, and on the data of reference [14].

\section{APPENDIX A}

Calculation of the number of trees. - We want to calculate the number $Q(S)$ of random walks with $S$ steps ( $S$ even) starting from the origin and returning to the origin, with the tree-like structure shown on figure $2 a$. A recurrence equation for $Q(S)$ can be constructed as shown on figure $2 c$ : We separate first the case where only two lines go to the origin, then the case of 4 lines, etc., obtaining

$$
\begin{aligned}
& Q(S)=z Q(S-2)+\frac{z^{2}}{2 !} \sum_{p} Q(S-2 p-2) Q(2 p)+ \\
& +\frac{z^{3}}{3 !} \sum_{p, q} Q(S-2 p-2 q-4) Q(2 p) Q(2 q) \\
& +\quad \ldots
\end{aligned}
$$


where the sums $\sum_{p}$ extend over all values leaving positive arguments for the $Q$ functions. Eq. (A.1) can be solved in detail through Laplace transforms. Here we shall simply consider the limit of large $S$, and look for solutions of the form

$$
Q(S)=\lambda^{S} \times \text { const }
$$

Inserting this in eq. (A.1) and dividing both sides by $Q(S)$ we obtain $1=\frac{z}{\lambda^{2}}+\frac{z^{2}}{2 !} \frac{1}{\lambda^{4}}+\frac{z^{3}}{3 !} \frac{1}{\lambda^{6}}+\cdots=\exp \left(\frac{z}{\lambda^{2}}\right)-1$

The solution is

$$
\lambda=(z / \ln 2)^{1 / 2} .
$$

The relative number of trees is thus

$$
P=\frac{Q(S)}{z^{S}}=\text { const }\left(\frac{1}{z \ln 2}\right)^{S / 2}
$$

as announced in eq. (2).

\section{References}

[1] Kramer, O., Greco, R., Ferry, J., J. Polym. Sci. 12 (1974) 2361.

[2] Kramer, O., Greco, R., Ferry, J., McDonel, E., to be published in J. Polym. Sci.

[3] De Gennes, P. G., J. Chem. Phys. 55 (1971) 572.

[4] Zilliox, J. G., Thèse Strasbourg (1970).

[5] SuzuKI, R., Thèse Strasbourg (1970).

[6] The importance of dangling chains in gels has been pointed out in particular by FERRY, J. D., Viscoelastic properties of polymers, 2nd ed. (Wiley, N. Y.) 1970.

[7] Berry, G. C., Fox, T. G., Adv. Polym. Sci. 5 (1968) 261.

[8] Kraus, G., Gruver, J. T., J. Polym. Sci. 8 (1970) 305.

[9] Fuлiмото, T. et al., Macromolecules 3 (1970) 57.
[10] Mendelson, R. et al., J. Polym. Sci. 8 (1970) 105, 127.

[11] Masuda, T. et al., Macromolecules 4 (1971) 763; Polym. J. 3 (1972) 92.

[12] Pannell, J., Polymer 13 (1972) 1.

[13] UtraCKI, L., ROOVERS, J., Macromolecules 6 (1973) 366.

[14] Graessley, W., Masuda, T., Roovers, J., to be published.

[15] For a general review of entanglement effects see : GRAESSLEY, W. W., Adv. Polym. Sci. 16 (Springer 1974).

[16] Edwards, S. F., Grant, J., J. Phys. A6 (1973) 1169 ; J. Phys. A6 (1973) 1186.

[17] Dol, M., Chem. Phys. Lett. 26 (1974) 269.

[18] Rouse, P. E'. J. Chem. Phys. 48 (1953) 1272.

[19] Сномpff, A. J., J. Chem. Phys. 53 (1970) 1566. 